\title{
Autophagy attenuates particulate matter 2.5-induced damage in HaCaT cells
}

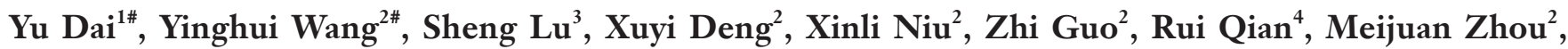 \\ Xuebiao Peng ${ }^{1}$
}

${ }^{1}$ Department of Dermatology, Nanfang Hospital, Southern Medical University, Guangzhou, China; ${ }^{2}$ Department of Radiation Medicine, Guangdong Provincial Key Laboratory of Tropical Disease Research, School of Public Health, Southern Medical University, Guangzhou, China; ${ }^{3}$ The First School of Clinical Medicine, Southern Medical University, Guangzhou, China; ${ }^{4}$ Department of General Surgery, Nanfang Hospital, Southern Medical University, Guangzhou, China

Contributions: (I) Conception and design: M Zhou, X Peng; (II) Administrative support: X Peng; (III) Provision of study materials or patients: M Zhou; (IV) Collection and assembly of data: Y Dai, Y Wang, S Lu, X Deng, X Niu, Z Guo, R Qian; (V) Data analysis and interpretation: Y Dai, Y Wang; (VI) Manuscript writing: All authors; (VII) Final approval of manuscript: All authors.

\#These authors contributed equally to this work and share first authorship.

Correspondence to: Meijuan Zhou. Department of Radiation Medicine, Guangdong Provincial Key Laboratory of Tropical Disease Research, School of Public Health, Southern Medical University, 1063 Jingxi Street, Guangzhou 510515, China. Email: 1kzmj@smu.edu.cn; Xuebiao Peng. Department of Dermatology, Nanfang Hospital, Southern Medical University, 1838 Guangzhou Avenue North, Guangzhou 510515, China. Email: pengxuebiao@126.com.

Background: Keratinocyte is a key component of the skin barrier and maintains skin homeostasis. As an environmental pathogenic factor, PM2.5 can cause epidermal cell damage, but the mechanism remains to be elucidated. The present study aimed to evaluate the effect caused by PM2.5 in HaCaT cells and investigate the underlying mechanisms.

Methods: HaCaT cells were treated with PM2.5 for $12 \mathrm{~h}$ or $24 \mathrm{~h}$, either alone or combined with UVB irradiation. A Cell Counting Kit (CCK-8) assay was carried out to detect the effect of PM2.5 on HaCaT cell viability. Flow cytometry, Western Blot, and AO staining were employed to detect the changes of apoptosis and autophagy. The changes of cytotoxicity and apoptosis in HaCaT cells were analyzed by CCK-8 and flow cytometry after pretreatment with autophagy inhibitor 3-MA.

Results: The results showed that PM2.5 induced cytotoxicity by increasing cell apoptosis and activating autophagy. Apoptosis was determined to be increased significantly after autophagy inhibition. Moreover, solar radiation intensified PM2.5-induced damage in HaCaT cells, which further enhanced the autophagy. However, there was no significant difference in apoptosis after inhibition of autophagy in combined treatment.

Conclusions: Our data reveals that PM2.5 induces damage in HaCaT cells, and autophagy plays a protective role to promote cell survival.

Keywords: Autophagy; PM2.5; HaCaT; apoptosis; cytotoxicity

Submitted Apr 07, 2021. Accepted for publication Jun 02, 2021.

doi: 10.21037/atm-21-2146

View this article at: https://dx.doi.org/10.21037/atm-21-2146

\section{Introduction}

Particulate matter (PM) comes from coal combustion and diesel vehicle exhaust, and is the primary pollutant effecting urban air quality in China (1). PM2.5 has an aerodynamic diameter less than 2.5 microns and is a mixture of many harmful ingredients, including carbon, organic chemicals, transition metals, and free radicals. In recent years, PM2.5 
has attracted the attention of many researchers and epidemiological studies have shown that it is a public health threat and closely related to mortality (2). According to a systematic analysis for the Global Burden of Disease Study 2017, ambient PM pollution accounted for $5.25 \%$ of global deaths and a total of 2.94 million worldwide, making it the 8th most common risk for death (3). Previous studies have suggested that PM2.5 exposure causes disease of several systems including the respiratory (4-6), cardiovascular $(7,8)$, reproductive $(9,10)$, and nervous systems $(11,12)$. Some researchers have shown that in industrial urban areas, acute PM2.5 exposure can exacerbate atopic dermatitis (13) and allergic dermatitis (14) among young children. A timestratified case-crossover analysis revealed that exposure to air pollution exacerbates inflammatory skin diseases, and there is a positive association between air pollution and hospital outpatient visits for eczema and dermatitis among the elderly and women (15). All the above studies have shown a close relationship between PM2.5 and skin damage. Thus, it is of interest to investigate the underlying mechanism of PM2.5-mediated skin injury.

The stratum corneum is the outermost layer of the skin and the first barrier for the body to resist environmental hazards by forming a crucial interface between the human body and the atmosphere $(16,17)$. PM2.5 can attach to the skin surface, or penetrate into skin transdermally or through hair follicles, causing toxic reactions in partial skin and distal organs (18). Hence, the interaction between PM2.5 and the skin barrier makes the underlying mechanism of skin lesions an important consideration when evaluating the skin toxicity of PM2.5. Ambient PM2.5 exposure reduced the viability of keratinocytes (19) and activated inflammatory factors including IL- 6 and TNF- $\alpha$, and all of the above processes led to inflammation in skin tissue (20). Dong et al. determined that oxidative stress is involved in the process of skin inflammatory and aggravates atopic dermatitis (21). Other researchers have found high levels of TNF induced by PM2.5 decreased the expression of filaggrin (FLG) and caused dysfunction of the skin barrier (22). PM2.5 pollution also accelerated skin senescence via aggregation and proteolytic degradation of serum lipoproteins, including high-density lipoprotein (HDL) and low-density lipoprotein (LDL) (23). One study revealed that PM2.5 and UVB, the major environmental pathogenic factor for dermal damage, could cause damage in epidermal cells and increase keratinocytes apoptosis. However, the effect and mechanism of PM2.5-induced damage in keratinocytes remains to be further explored (24).
Autophagy is a scavenging process of protein aggregates and damaged organelles mediated by lysosomes. Under physiological conditions, autophagy is continuously kept at a basal level to maintain protein homeostasis (proteostasis) and the stability of the intracellular environment (25). Autophagy is rapidly upregulated in response to limited stress stimuli such as hypoxia and nutrient deprivation, thus promoting cell survival (26). However, when the stress is severe or persistent, excessive autophagy is induced, leading to abnormal cell function and ultimately promoting cell senescence and death (27). Whether autophagy produces adaptive responses to cell survival or initiates programmed cell death depends on the stage of cell growth and the changes in extracellular environment $(28,29)$. However, the relationship between autophagy and PM2.5 has not been adequately studied. In human endothelial cells, autophagy confers a protective role against PM2.5-induced apoptosis to promote cell survival (30). PM2.5 activated the PINK1/Parking signal pathway via oxidative stress to induce mitophagy, which stimulated HSCs and led to liver fibrosis (31), and in human bronchial epithelium cells, PM2.5 increased the expression of NOS2 and NO and subsequent excessive autophagy, leading to cell death (32), while resveratrol attenuates the oxidative injury through autophagy deregulation (33) Therefore, it is necessary to address the effect of autophagy on cell fate in PM2.5 induced skin damage.

In this study, we confirmed for the first time that PM2.5 and/or UVB exposure could cause keratinocyte damage, and that blocking autophagy augmented the cytotoxicity induced by PM2.5, in which autophagy plays a protective role. We hope that this study can provide evidence for the treatment of skin disease related to environmental pollution.

We present the following article in accordance with the MDAR reporting checklist (available at https://dx.doi. org/10.21037/atm-21-2146).

\section{Methods}

\section{Preparation of PM2.5}

PM2.5 was collected using a Thermo Anderson G-2.5 air sampler (Thermo Scientific, Franklin, MA, USA) between November 2018 and January 2019 from the roof of a building in Baiyun District, Guangzhou. Glass microfiber filters were cut into $1 \mathrm{~cm}^{2}$, suspended in ultrapure water, and sonicated for $4 \times 30$ min with a sonicator (Shumei, Kunshan, China). The PM2.5 suspensions were then filtered with a 
300-meshes aseptic sieve to remove the quartz fibre as this may have affected the experimental results. The filtrate was treated by vacuum freeze drying, and lyophilized ingredients were weighted and stored at $-20^{\circ} \mathrm{C}$.

\section{Cell culture}

The human keratinocyte cell line HaCaT (CellCook Biotech Co. Ltd, Guangzhou, China) was cultured at $37{ }^{\circ} \mathrm{C}$ in a $5 \% \mathrm{CO}_{2}$ incubator. The cells were maintained in Dulbecco's Modified Eagle Medium (DMEM) (Gibco, Grand Island, NY, USA) with 10\% newborn bovine serum (NBS) (Invitrogen, Carlsbad, CA, USA) and 1\% antibiotic (100 $\mathrm{g} / \mathrm{mL}$ streptomycin, $100 \mathrm{U} / \mathrm{mL}$ penicillin) (Gibco, Grand Island, NY, USA). HaCaT cells were cultured for 24 h with DMEM containing 10\% NBS, then pretreated with 3-Methyladenine (3-MA) (Selleckchem, Houston, TX, USA) for $2 \mathrm{~h}$, and further exposed to PM2.5 and UVB for $12 / 24 \mathrm{~h}$.

\section{Cell viability}

$\mathrm{HaCaT}$ were seeded in 96-well plates by culture medium for 24 h. PM2.5 in PBS was added to the wells to ensure a final concentration of $100 \mu \mathrm{g} / \mathrm{mL}$. After $12 / 24 \mathrm{~h}$, Cell Counting Kit-8 (CCK-8) (MCE, NJ, USA) was added to the wells with a pipet, and the optical density of the cells was measured by spectrophotometer to assess viability.

\section{Apoptosis}

The cells were seeded in 6-well plates for $24 \mathrm{~h}$, and after treatment, were digested with trypsin, centrifuged at $500 \mathrm{~g}$ for $5 \mathrm{~min}$ and resuspended with $1 \times$ binding buffer. PI and FITIC were added to the suspension and reacted for 5 min protected from light. Cell apoptosis was measured and quantified using a flow cytometer (BD, Grand Island, NY, USA).

\section{Western blot}

$\mathrm{HaCa} \mathrm{T}$ was seeded in $6 \mathrm{~cm}$ dishes in DMEM and treated $24 \mathrm{~h}$ later. After processing, the cells were washed twice with PBS and lysed on ice for $40 \mathrm{~min}$ in RIPA buffer (Beyotime, Shanghai, China), which contained protease inhibitor cock (PIC) (Sigma Aldrich, Boston, MA, USA) and $1 \mathrm{mM}$ phenylmethylsulfonyl fluoride (PMSF) (Sigma Aldrich, Boston, MA, USA). The lysate was centrifuged at
$12,000 \mathrm{rpm}$ for 10 minutes and the supernatant was stored at $-80{ }^{\circ} \mathrm{C}$. Samples were quantified using a BCA quantification kit (Beyotime, Shanghai, China), and equal amounts of protein were run on $15 \%$ separating gel and $5 \%$ stacking gel. Proteins were transferred onto a $0.45 \mu \mathrm{m}$ polyvinylidene difluoride (PVDF) membrane (Thermo Fisher Scientific, Waltham, MA, USA) and the membrane was blocked with $5 \%$ skim milk in $1 \times$ TBST. Antibodies against LC3 I/II (1:1,000, Abcam, Cambridge, UK), SQSTM1/p62 (1:1,000, Abcam, Cambridge, UK), and $\beta$-actin (1:5,000, Santa Cruz Biotechnology, Santa Cruz, CA, USA) were incubated overnight at $4{ }^{\circ} \mathrm{C}$. The membranes were then washed with $1 \times$ TBST for $10 \mathrm{~min}$ three times, then incubated with horseradish peroxidase conjugated anti-mouse $(1: 5,000)$ or anti-rabbit $(1: 5,000)$ secondary antibodies at room temperature for $1 \mathrm{~h}$. The blots were imaged with Luminata Forte Western HRP substrate (Millipore, Boston, MA, USA).

\section{Detection of intracellular ROS}

To detect the intracellular ROS in $\mathrm{HaCaT}$, the cells were seeded in 6-well plates and cultured for $24 \mathrm{~h}$, then treated with $100 \mu \mathrm{g} / \mathrm{mL}$ PM2.5 and/or $30 \mathrm{~J} / \mathrm{m}^{2}$ UVB. Twelve or 24 hours later, the cells were washed twice using PBS, digested with trypsin, centrifuged at $500 \mathrm{~g}$ for $5 \mathrm{~min}$, and resuspended with DMEM, after which a 2',7'-dichlorodih ydrofluorescein diacetate (DCF-DA) (Beyotime, Shanghai, China) solution was added to the cells. The cells were incubated at $37{ }^{\circ} \mathrm{C}$ for $20 \mathrm{~min}$ and during the incubation period, were mixed upside down every $2-3$ minutes and then washed with DMEM three times. DCF fluorescence was measured and quantified using a flow cytometer (BD, Grand Island, NY, USA).

\section{AO staining}

The $\mathrm{HaCaT}$ was seeded in 24-well plates for $24 \mathrm{~h}$, and after treatment, the cells were twice washed with $1 \times$ wash buffer. Acridine orange (Solarbio, Wuhan, China) was added at a final concentration of $1 \mathrm{mg} / \mathrm{mL}$ for 15 minutes, dried at room temperature without light, and detected with a fluorescence microscope (OLYMPUS, Tokyo, Japan).

\section{Statistical analyses}

Graphical visualization was implemented with GraphPad Prism 5.0 (GraphPad Software) and statistical analysis was 
carried out by SPSS 20.0 software. All data were expressed as mean \pm standard deviation (mean $\pm \mathrm{SD}$ ) and $\mathrm{P}<0.05$ was considered statistically significant.

\section{Results}

\section{PM2.5 induces cytotoxicity in HaCaT cells}

To evaluate the damage induced by PM2.5, the survival rate of $\mathrm{HaCaT}$ cells was assessed by CCK- 8 . The cytotoxic effects of PM2.5 were detected in human keratinocytes exposed to $0,10,25,50,100,200,400$, and $500 \mu \mathrm{g} / \mathrm{mL}$ PM2.5 for $24 \mathrm{~h}$, and cell viability decreased in a dosedependent manner (Figure 1A). We further detected the effect of PM2.5 on the proliferation of $\mathrm{HaCaT}$ and found that PM2.5 could inhibit the proliferation of keratinocyte cells significantly in a time-dependent manner (Figure 1B). We then explored the type of cell death after PM2.5 treatment. Human keratinocytes cultured after PM2.5 exposure were assessed by flow cytometry, and apoptosis at $24 \mathrm{~h}$ was significantly higher than $12 \mathrm{~h}$, indicating that the increase in PM2.5 promoted apoptosis was time-dependent (Figure 1C). Western blot revealed an obvious induction of PARP in HaCaT cells (Figure 1D). Taken together, these data indicate that PM2.5 affects cell survival by inducing apoptosis.

\section{Protective autophagy is triggered by PM2.5 treatment}

Autophagy is crucial among factors that determine the fate of cells. We next investigated whether PM2.5 could induce autophagy in $\mathrm{HaCaT}$ cells. AO staining results depicted a significant accumulation of autophagosomes in HaCaT exposed to PM2.5, compared to levels in cells not exposed to PM2.5, and were greater in the $24 \mathrm{~h}$ treatment group compared with the $12 \mathrm{~h}$ group (Figure $2 A$ ). The transition from LC3-I to LC3-II, the processed form of LC3, represents the activation of autophagy, and our results showed PM2.5 upregulated the expression of SQSTM1/p62 and LC3-II in a time-dependent manner (Figure 2B). The above results indicated that PM2.5 can induce autophagy in $\mathrm{HaCaT}$ cells. We next discussed the possible mechanism to this process, and accumulated evidence indicates that PM2.5 induces oxidative stress by generating ROS, leading to autophagy. Figure $2 C$ shows that PM2.5 generated higher levels of ROS in $\mathrm{HaCaT}$ cells $24 \mathrm{~h}$ after treatment, compared to cells treated after $12 \mathrm{~h}$. To clarify the relation between autophagy and apoptosis induced by PM2.5,
3-MA, an autophagy inhibitor, was used for subsequent experiments. This showed cell viability decreased after the pretreatment of 3-MA, and the effect was more significant at $24 \mathrm{~h}$ (Figure 2D). We further investigated the effect of 3-MA pretreatment on apoptosis in $\mathrm{HaCaT}$ cells, and consistent with the previous results, 3-MA aggravated apoptosis in a time-dependent manner (Figure 2E), suggesting autophagy played a protective role against apoptosis in response to PM2.5 in HaCaT cells.

\section{UVB irradiation aggravates PM2.5-induced apoptosis in HaCaT cells}

The factors that cause skin diseases are often multiple, and we selected UVB to study the damage caused by its combination with PM2.5 on skin cells. While UVB exposure is unavoidable, in excess it can induce erythema, ageing, and eventually, skin cancer (34). To determine whether UVB aggravated PM2.5-induced damage to HaCaT, cell viability was assessed by CCK- 8 . The results showed that cell viability was decreased in cells treated by PM2.5 and UVB after 12 and $24 \mathrm{~h}$, respectively, and the decrease was more obvious after combined treatment (Figure $3 A$ ). In addition, consistent with the results of cytotoxic effects, apoptosis rates were significantly increased in cells treated by PM2.5 combined with UVB (Figure 3B). Notably, the cytotoxicity induced by UVB was significantly greater than that by PM2.5 after $24 \mathrm{~h}$, compared to $12 \mathrm{~h}$. We verified the above results by Western blot and found that PM2.5 and/or UVB could induce apoptosis (Figure 3C), and that apoptosis-related protein, PARP, was increased after PM2.5 and UVB treatment. These observations suggest that UVB aggravated PM2.5-induced apoptosis.

\section{Autophagy induced by PM2.5 treatment is enhanced by ultraviolet $B$ exposure}

To explore the related effects of PM2.5 and UVB exposure in keratinocytes, we analyzed intracellular autophagy levels. In cultured keratinocytes, PM2.5 and UVB triggered the accumulation of intracellular autophagic vacuoles indicative of autophagosome formation at 12 and $24 \mathrm{~h}$, as evidenced by AO staining. We found the red/green fluorescence ratio of the PM2.5 treatment group was fewer than that of the UVB treatment group, which was fewer than the PM2.5UVB treatment group, suggesting that UVB aggravated autophagy induced by PM2.5. In addition, the red/green fluorescence ratio per cell was increased at $24 \mathrm{~h}$, compared 


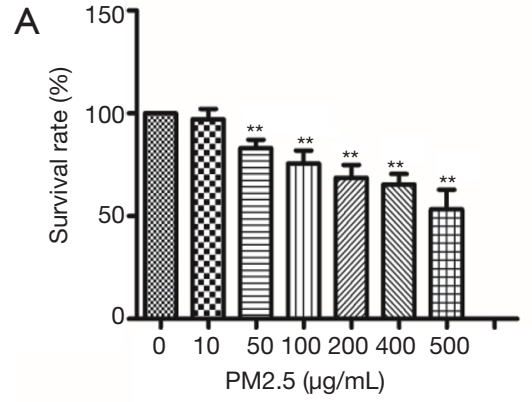

C
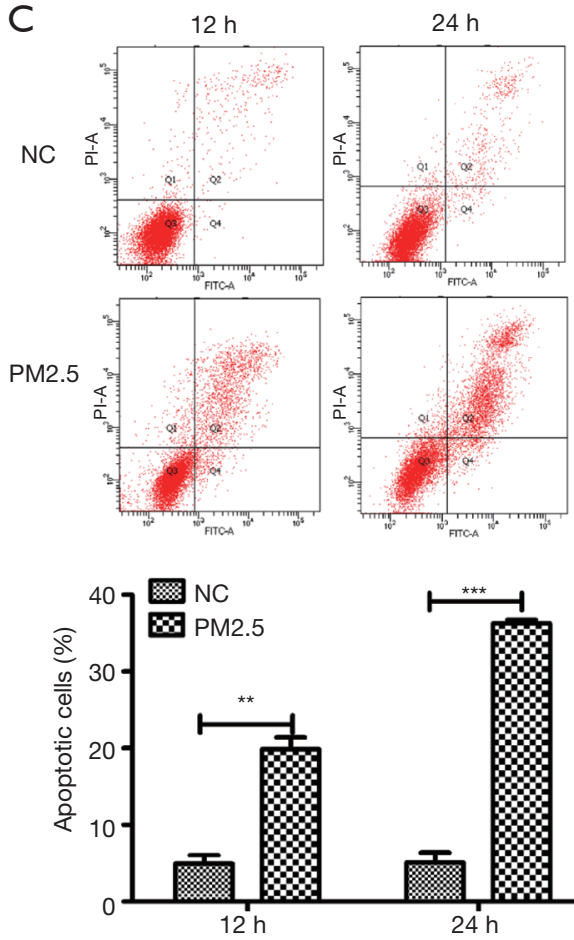

B

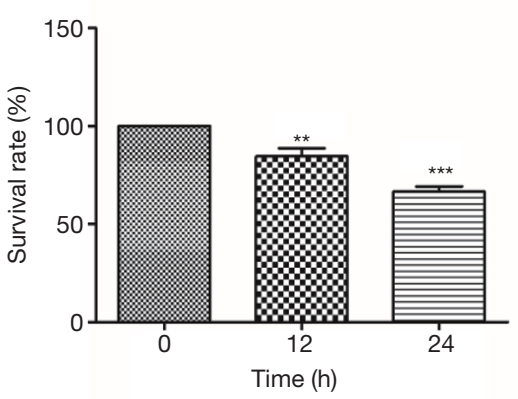

D

Pro-PARP

Cleaved-PARP

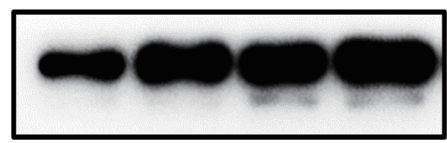

$\beta$-actin

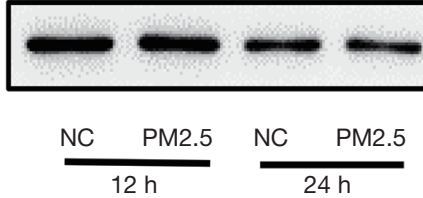

Figure 1 PM2.5 induces cytotoxicity in HaCaT cells. (A) HaCaT cells were treated with PM2.5 (0, 10, 50, 100, 200, 400, and 500 $\mu$ g/mL) for $24 \mathrm{~h}$ and cell viability was determined by CCK-8; (B) HaCaT cells were treated with $100 \mu \mathrm{g} / \mathrm{mL} \mathrm{PM} 2.5$, and the cell viability was measured at 0, 12, and $24 \mathrm{~h}$; (C) cell apoptosis was detected by Annexin V-FITC/PI double staining and flow cytometry; (D) apoptosisrelated protein was detected by western blot. Each experiment was performed in triplicate and data are presented as mean \pm SD. One-way ANOVA and Dunnett's Multiple comparison test were used to analyze the data $\left.{ }^{* *} \mathrm{P}<0.01,{ }^{* * *} \mathrm{P}<0.001\right)$.

with $12 \mathrm{~h}$, indicating autophagy increased in a timedependent manner during this process (Figure 4A). These results were then verified by western blot. Compared with the single treatment group, PM2.5 combined with UVB significantly up-regulated the expression of LC3-II and down-regulated SQSTM1/p62. Interestingly, PM2.5 treatment and UVB treatment showed completely opposite trends in SQSTM1/p62 expression. Moreover, these results were more pronounced in the $24 \mathrm{~h}$ treatment group than in the $12 \mathrm{~h}$ treatment group (Figure $4 B$ ). These phenomena may be related to different mechanisms of autophagy. To investigate the potential role of oxidative stress induced by PM2.5 and/or UVB, we detected ROS generation in HaCaT at different time points by flow cytometry (Figure $4 C$ ). The generation of ROS in the UVB treatment group was more than that in the PM2.5 treatment group, but less than that in the PM2.5-UVB treatment group, and the results at different time points were consistent, indicating that UVB increased ROS induced by PM2.5. In conclusion, the above data showed that UVB could enhance PM2.5-induced 


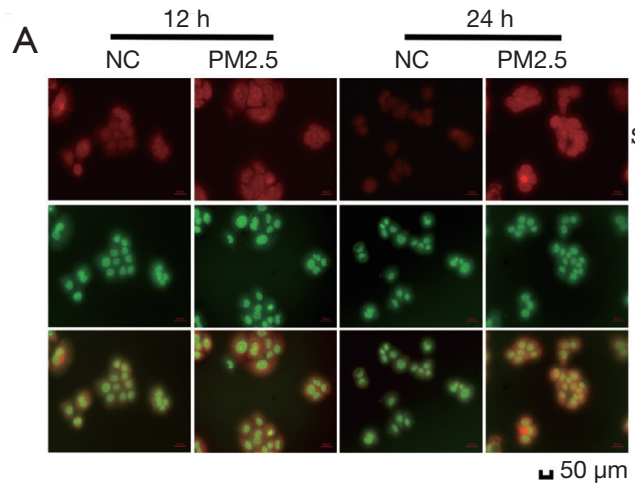

B
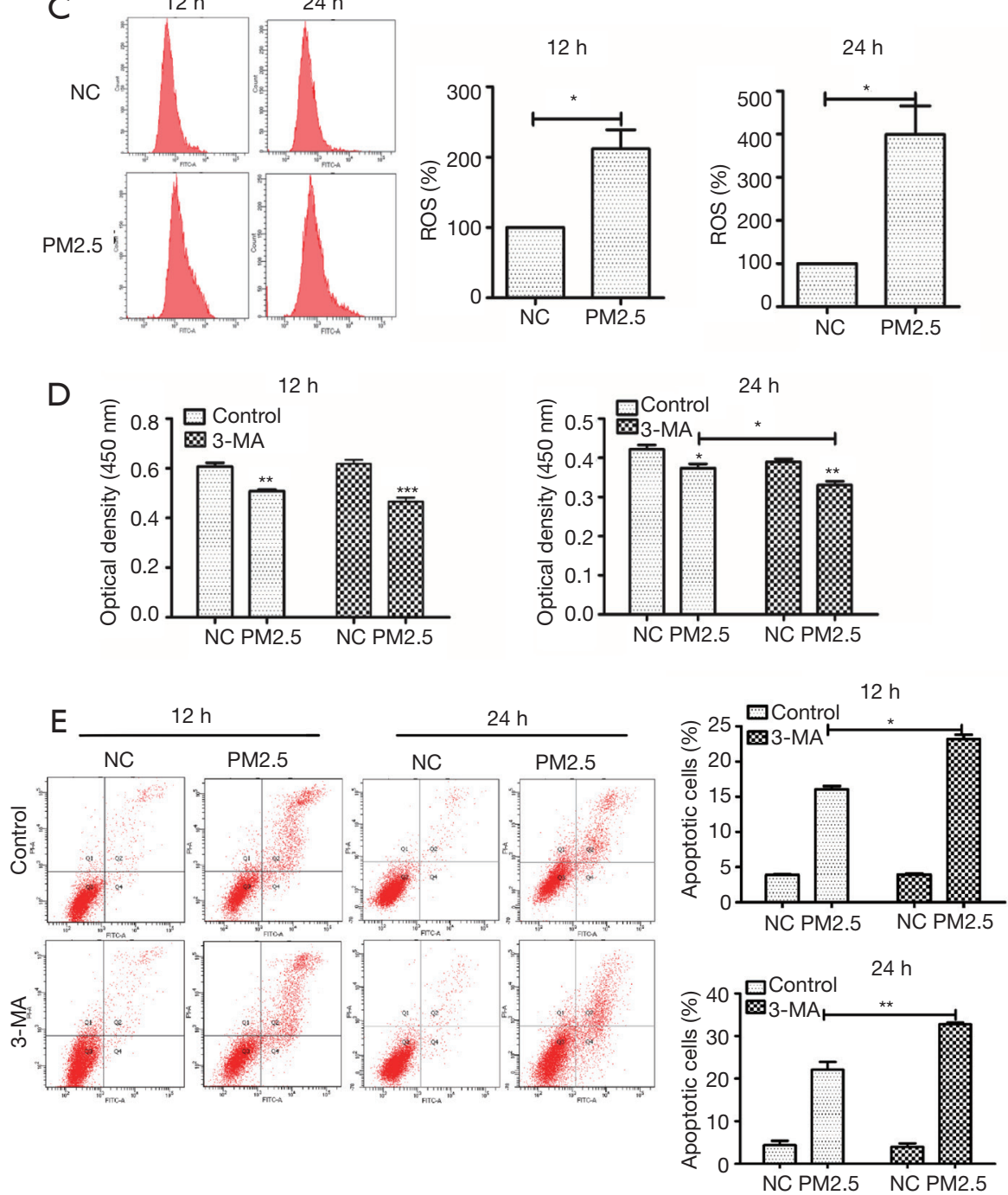

Figure 2 Autophagy plays a protective role in $\mathrm{HaCaT}$. (A) AO staining results depict a significant accumulation of autophagosomes in $\mathrm{HaCaT}$ exposed to PM2.5. (B) Autophagy-related proteins were detected by western blot. (C) ROS generation was assessed by the H2DCFDA assay at 12 and $24 \mathrm{~h}$. Cells were pre-treated with 3-MA for $2 \mathrm{~h}$, cell viability (D) was detected by CCK-8, and apoptosis (E) was detected by Annexin V-FITC/PI double staining and flow cytometry. Magnification: 200x. Each experiment was performed in triplicate and data are presented as mean $\pm \mathrm{SD}$. One-way ANOVA and Dunnett's Multiple comparison test were used to analyze the data ${ }^{*} \mathrm{P}<0.05$, $\left.{ }^{* *} \mathrm{P}<0.01\right)$. 

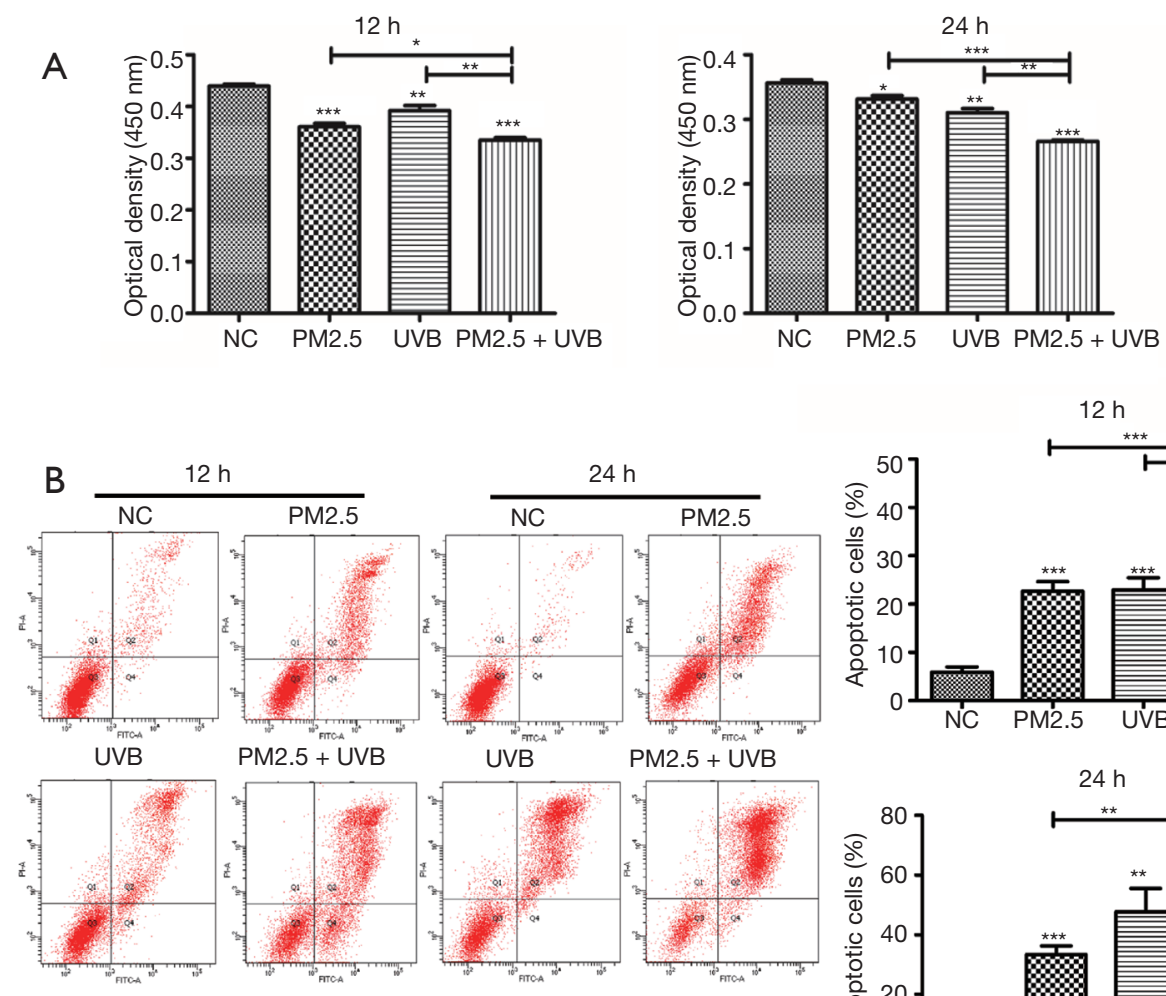

C
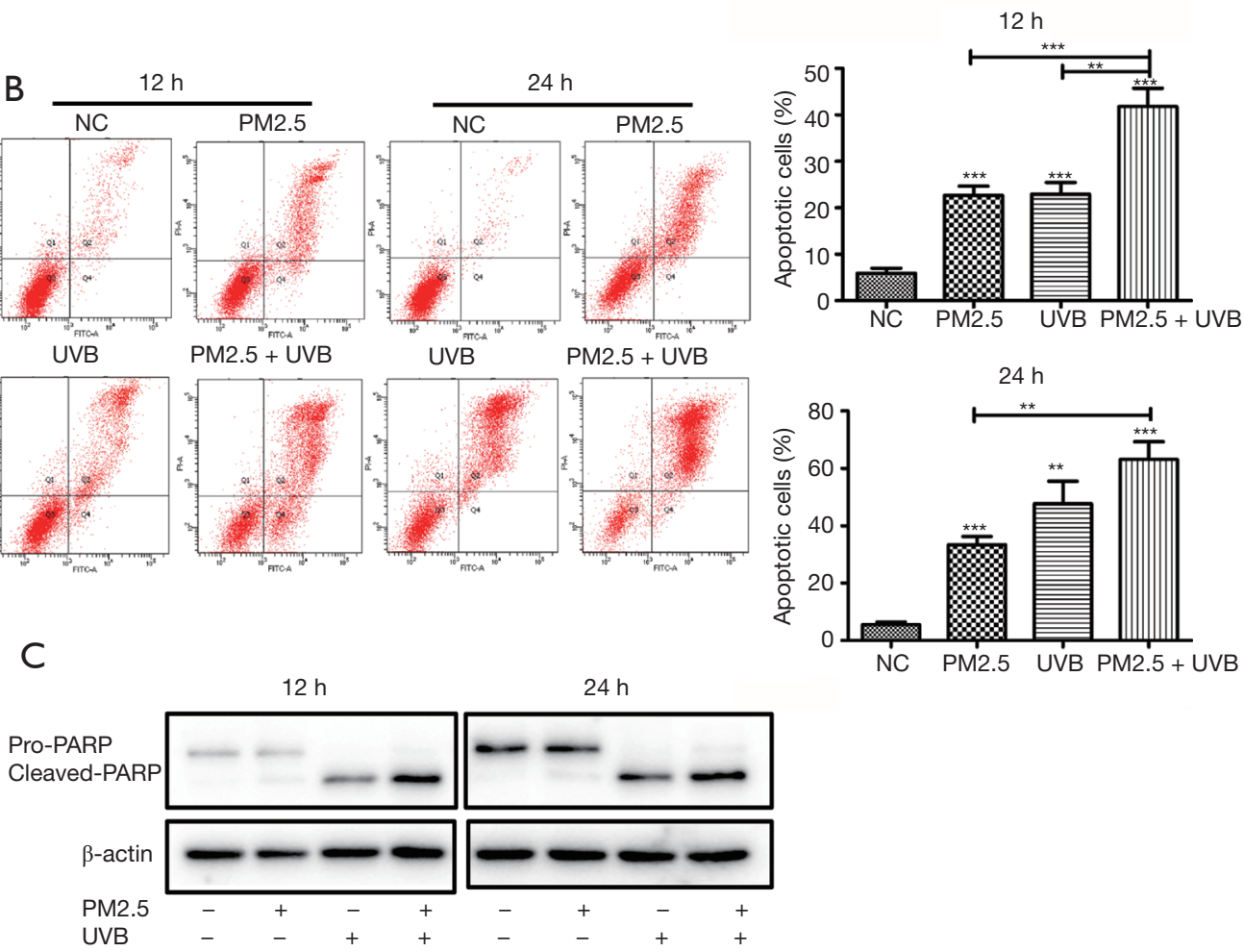

Figure 3 Cell death increases after PM2.5 combined with UVB. (A) Cell viability decreased after PM2.5 combined with UVB; (B,C) apoptosis increased after PM2.5 combined with UVB. Each experiment was performed in triplicate and data are presented as mean \pm SD. One-way ANOVA and Dunnett's Multiple comparison test were used to analyze the data $\left({ }^{*} \mathrm{P}<0.05,{ }^{* *} \mathrm{P}<0.01,{ }^{* * *} \mathrm{P}<0.001\right)$.

autophagy in $\mathrm{HaCaT}$.

\section{Discussion}

The skin is the largest organ of the human body and the first barrier to resist environmental pollutants. PM2.5 easily absorbs organic compounds in the air, such as PAHs, due to its large specific surface area and strong enrichment. Long-term exposure to high concentrations of PM2.5 can lead to skin ageing, local pigmentation, increased pigmentation spots, and nasolabial sulci (35). PM2.5 can damage the integrity of the skin barrier by destroying the involucrin, including loricrin (LOR), involucrin (ILV), and filaggrin (FLG), which play an important role in skin differentiation (36). Our study found that in keratinocytes, PM2.5 induced cytotoxicity in a time-dependent and concentration-dependent manner. Recent studies indicate that environmental factors, including PM and solar UVR, contribute to the development of facial lentigines (37). A meta-analysis demonstrated that smoking increased the risk of cutaneous squamous cell carcinoma, the main risk factor of which is UV exposure (38). Therefore, we selected UVB as the treatment factor for the experiment, and discovered that UVB aggravated cytotoxicity induced by PM2.5. The 

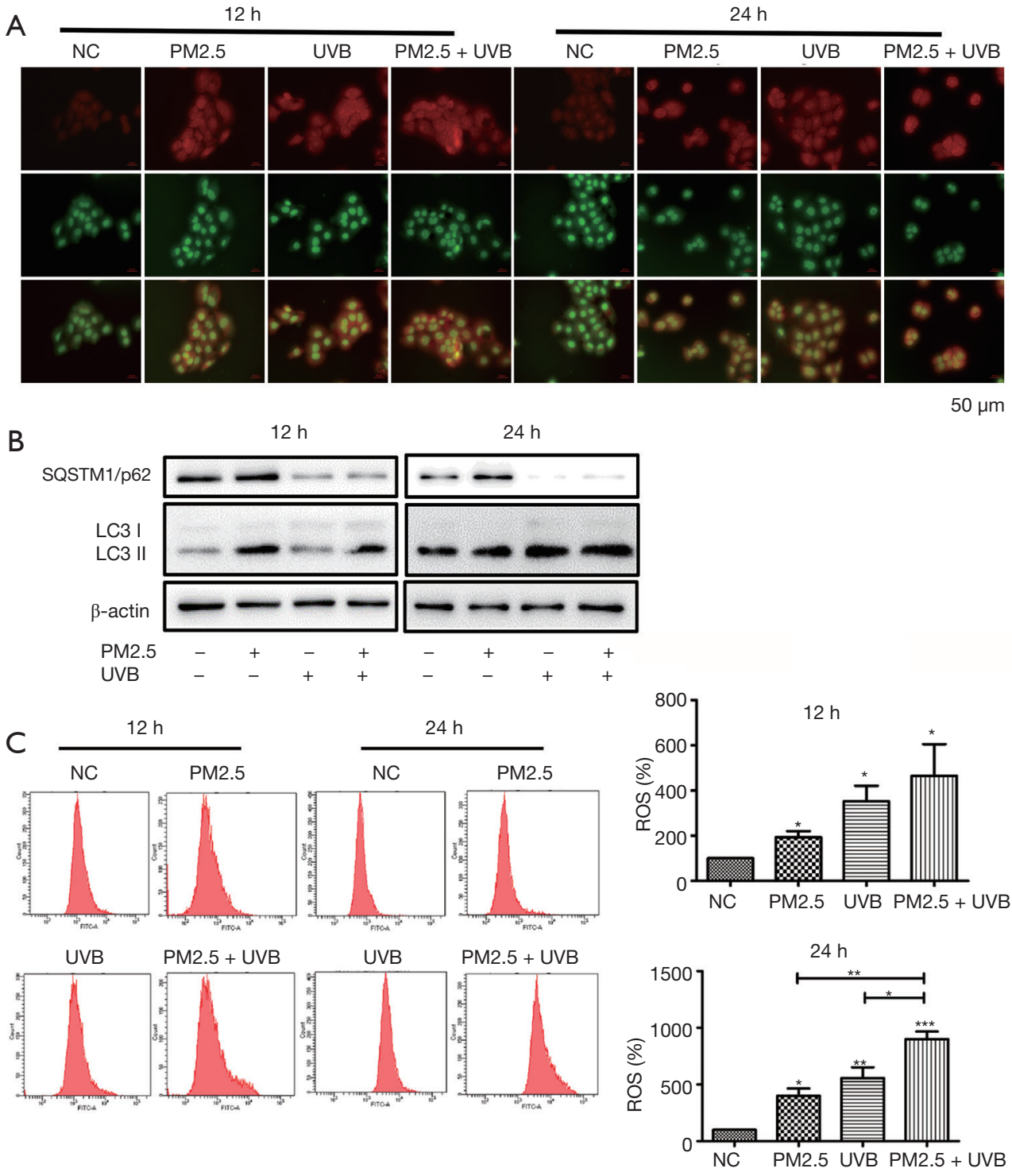

Figure 4 UVB enhances PM2.5 induced autophagy. (A,B) Autophagy was enhanced after PM2.5 combined with UVB. (C) ROS production increased after PM2.5 combined with UVB. Magnification: 200x. Each experiment was performed in triplicate and data are presented as mean \pm SD. One-way ANOVA and Dunnett's Multiple comparison test were used to analyze the data $\left({ }^{*} \mathrm{P}<0.05,{ }^{* *} \mathrm{P}<0.01,{ }^{* * *} \mathrm{P}<0.001\right)$.

mechanisms by which PM2.5 and UVB lead to cell death are unclear. Inflammation, oxidative stress, and metabolic damage are the most probable mechanisms accounting for skin disease associated with pollution, and these stresses can be amplified by the harmful synergies between pollution and sunlight (39). PM and some of the PAHs could absorb UV and generate reactive oxygen such as single oxygen, which resulted in lipid peroxidation, DNA damage, and finally, cell death (40). At present, although the mechanism of skin damage induced by PM2.5 and UVB remains unclear, we speculate that autophagy may be involved in this process.

Previous studies have shown that PM2.5 damages skin cells by inducing oxidative stress, leading to dysfunction in cellular organelles including mitochondria, ER, and lysosomes (41). Oxidative stress is also a major feature of autophagy and can produce a large amount of ROS to destroy organelles. When organelles are damaged, cells remove or degrade the damaged organelles and protein macromolecules by activating autophagy to maintain the normal function and homeostasis of cells. Cells then initiate 
an autophagy reaction which leads to a series of changes when intracellular ROS accumulate in large quantities. ROS induces the production of AMP and ATP in cells, which can activate the AMPK pathway, inhibit the mTORC1 pathway, and induce autophagy initiation. Further, this can promote the transcription of Beclin1, SQSTM1/p62, and other autophagy genes by regulating the activity of transcription factors, such as $\mathrm{NF}-\kappa \mathrm{B}$. In this study, we revealed that PM2.5 can stimulate the increase of ROS in $\mathrm{HaCaT}$ cells and lead to the enhancement of autophagy, which is consistent with the results of previous studies. Compared with the control group, apoptosis increased obviously in the 3-MA pre-treated group, indicating that autophagy antagonized the lethal effect of apoptosis. In other words, autophagy played a protective role against apoptosis in response to $\mathrm{PM} 2.5$.

The process of autophagy is related to a variety of autophagy-related genes, including LC3, SQSTM1/p62, and Beclin1. LC3 enzymatically decomposes a small section of polypeptide to form LC3-I, which eventually binds with PE to transform into LC3-II. Therefore, the ratio of LC3-II/I can be used as a marker to estimate the level of autophagy. As an autophagy adaptor, SQSTM1/p62 plays an important role in DNA damage and oxidative stress. Studies have shown that an increase in LC3-II, the induction of Beclin1 expression, and a decrease in SQSTM1/p62 levels, are hallmarks of autophagosome accumulation and autophagic degradation, and for this reason, the levels of these specific autophagy related proteins were analyzed by western bolt assay. Our study found that the expression of LC3-II and SQSTM1/p62 were not consistent in PM2.5 and UVB treatment groups, indicating that PM2.5 and UVB induce autophagy in different ways. We speculate this may be related to the disruption of autophagy flow, and in subsequent experiments, will further explore the mechanism.

Taken together, our study detected that PM2.5 alone and combined with UVB treatment could induce apoptosis and autophagy in a time-dependent manner. Autophagy plays a protective role against apoptosis in response to PM2.5. We hope that our research can provide a theoretical basis for further studies investigation protection from environmental pollutants.

\section{Acknowledgments}

Funding: This work was supported by grants from the National Natural Science Foundation of China (Grant No. 31971167) and China Postdoctoral Science Foundation
(Grant No. 2018M640805).

\section{Footnote}

Reporting Checklist: The authors have completed the MDAR reporting checklist. Available at https://dx.doi. org/10.21037/atm-21-2146

Data Sharing Statement: Available at https://dx.doi. org/10.21037/atm-21-2146

Conflicts of Interest: All authors have completed the ICMJE uniform disclosure form (available at https://dx.doi. org/10.21037/atm-21-2146). The authors have no conflicts of interest to declare.

Ethical Statement: The authors are accountable for all aspects of the work in ensuring that questions related to the accuracy or integrity of any part of the work are appropriately investigated and resolved.

Open Access Statement: This is an Open Access article distributed in accordance with the Creative Commons Attribution-NonCommercial-NoDerivs 4.0 International License (CC BY-NC-ND 4.0), which permits the noncommercial replication and distribution of the article with the strict proviso that no changes or edits are made and the original work is properly cited (including links to both the formal publication through the relevant DOI and the license). See: https://creativecommons.org/licenses/by-nc-nd/4.0/.

\section{References}

1. Huang C, Wang Q, Wang S, et al. Air Pollution Prevention and Control Policy in China. Adv Exp Med Biol 2017;1017:243-61.

2. Wang Y, Shi L, Lee M, et al. Long-term Exposure to PM2.5 and Mortality Among Older Adults in the Southeastern US. Epidemiology 2017;28:207-14.

3. Stanaway JD, Afshin A, Gakidou E, et al. Global, regional, and national comparative risk assessment of 84 behavioural, environmental and occupational, and metabolic risks or clusters of risks for 195 countries and territories, 1990-2017: a systematic analysis for the Global Burden of Disease Study 2017. Lancet 2018;392:1923-94.

4. Polezer G, Tadano YS, Siqueira HV, et al. Assessing the impact of PM2.5 on respiratory disease using artificial neural networks. Environ Pollut 2018;235:394-403. 
5. Wu S, Ni Y, Li H, et al. Short-term exposure to high ambient air pollution increases airway inflammation and respiratory symptoms in chronic obstructive pulmonary disease patients in Beijing, China. Environ Int 2016;94:76-82.

6. Wang M, Aaron CP, Madrigano J, et al. Association Between Long-term Exposure to Ambient Air Pollution and Change in Quantitatively Assessed Emphysema and Lung Function. JAMA 2019;322:546-56.

7. Hayes RB, Lim C, Zhang Y, et al. PM2.5 air pollution and cause-specific cardiovascular disease mortality. Int J Epidemiol 2020;49:25-35.

8. Dabass A, Talbott EO, Venkat A, et al. Association of exposure to particulate matter (PM2.5) air pollution and biomarkers of cardiovascular disease risk in adult NHANES participants (2001-2008). Int J Hyg Environ Health 2016;219:301-10.

9. Lafuente R, Garcia-Blaquez N, Jacquemin B, et al. Outdoor air pollution and sperm quality. Fertil Steril 2016;106:880-96.

10. Guo T, Wang Y, Zhang H, et al. The association between ambient PM2.5 exposure and the risk of preterm birth in China: A retrospective cohort study. Sci Total Environ 2018;633:1453-9.

11. Shou Y, Huang Y, Zhu X, et al. A review of the possible associations between ambient PM2.5 exposures and the development of Alzheimer's disease. Ecotoxicol Environ Saf 2019;174:344-52.

12. Kioumourtzoglou MA, Schwartz JD, Weisskopf MG, et al. Long-term PM2.5 Exposure and Neurological Hospital Admissions in the Northeastern United States. Environ Health Perspect 2016;124:23-9.

13. Oh I, Lee J, Ahn K, et al. Association between particulate matter concentration and symptoms of atopic dermatitis in children living in an industrial urban area of South Korea. Environmental Research 2018;160:462-8.

14. Morgenstern V, Zutavern A, Cyrys J, et al. Atopic diseases, allergic sensitization, and exposure to traffic-related air pollution in children. Am J Respir Crit Care Med 2008;177:1331-7.

15. Guo Q, Liang F, Tian L, et al. Ambient air pollution and the hospital outpatient visits for eczema and dermatitis in Beijing: a time-stratified case-crossover analysis. Environ Sci Process Impacts 2019;21:163-73.

16. Zhang D, Lu C, Yu Z, et al. Echinacoside Alleviates UVB Irradiation-Mediated Skin Damage via Inhibition of Oxidative Stress, DNA Damage, and Apoptosis. Oxid Med Cell Longev 2017;2017:6851464.
17. Bäsler K, Bergmann S, Heisig M, et al. The role of tight junctions in skin barrier function and dermal absorption. J Control Release 2016;242:105-18.

18. Puri P, Nandar SK, Kathuria S, et al. Effects of air pollution on the skin: A review. Indian J Dermatol Venereol Leprol 2017;83:415-23.

19. Suo D, Zeng S, Zhang J, et al. PM2.5 induces apoptosis, oxidative stress injury and melanin metabolic disorder in human melanocytes. Exp Ther Med 2020;19:3227-38.

20. Fernando IPS, Jayawardena TU, Sanjeewa KKA, et al. Anti-inflammatory potential of alginic acid from Sargassum horneri against urban aerosol-induced inflammatory responses in keratinocytes and macrophages. Ecotoxicol Environ Saf 2018;160:24-31.

21. Dong YM, Liao LY, Li L, et al. Skin inflammation induced by ambient particulate matter in China. Sci Total Environ 2019;682:364-73.

22. Kim BE, Kim J, Goleva E, et al. Particulate matter causes skin barrier dysfunction. JCI Insight 2021;6:e145185.

23. Kim JY, Lee EY, Choi I, et al. Effects of the Particulate Matter(2).(5) (PM(2).(5)) on Lipoprotein Metabolism, Uptake and Degradation, and Embryo Toxicity. Mol Cells 2015;38:1096-104.

24. Zhen AX, Piao MJ, Hyun YJ, et al. Purpurogallin Protects Keratinocytes from Damage and Apoptosis Induced by Ultraviolet B Radiation and Particulate Matter 2.5. Biomol Ther (Seoul) 2019;27:395-403.

25. Lahiri V, Hawkins WD, Klionsky DJ. Watch What You (Self-) Eat: Autophagic Mechanisms that Modulate Metabolism. Cell Metab 2019;29:803-26.

26. Dikic I, Elazar Z. Mechanism and medical implications of mammalian autophagy. Nature Reviews Molecular Cell Biology 2018;19:349-64.

27. Bialik S, Dasari SK, Kimchi A. Autophagy-dependent cell death - where, how and why a cell eats itself to death. J Cell Sci 2018;131:jcs215152.

28. Xiao M, Li L, Li C, et al. Role of autophagy and apoptosis in wound tissue of deep second-degree burn in rats. Acad Emerg Med 2014;21:383-91.

29. Eisenberg-Lerner A, Bialik S, Simon HU, et al. Life and death partners: apoptosis, autophagy and the cross-talk between them. Cell Death Differ 2009;16:966-75.

30. Wang Y, Tang M. PM2.5 induces autophagy and apoptosis through endoplasmic reticulum stress in human endothelial cells. Sci Total Environ 2020;710:136397.

31. Qiu YN, Wang GH, Zhou F, et al. PM2.5 induces liver fibrosis via triggering ROS-mediated mitophagy. Ecotoxicol Environ Saf 2019;167:178-87. 
32. Zhu XM, Wang Q, Xing WW, et al. PM2.5 induces autophagy-mediated cell death via NOS2 signaling in human bronchial epithelium cells. Int J Biol Sci 2018;14:557-64.

33. Li Y, Qian W, Wang D, et al. Resveratrol relieves particulate matter (mean diameter $<2.5$ mum)-induced oxidative injury of lung cells through attenuation of autophagy deregulation. J Appl Toxicol 2018;38:1251-61.

34. Matsumura Y, Ananthaswamy HN. Toxic effects of ultraviolet radiation on the skin. Toxicol Appl Pharmacol 2004;195:298-308.

35. Ding A, Yang Y, Zhao Z, et al. Indoor PM2.5 exposure affects skin aging manifestation in a Chinese population. Sci Rep 2017;7:15329.

36. Li Q, Kang Z, Jiang S, et al. Effects of Ambient Fine Particles PM2.5 on Human HaCaT Cells. Int J Environ Res Public Health 2017;14:72.

Cite this article as: Dai Y, Wang Y, Lu S, Deng X, Niu X, Guo Z, Qian R, Zhou M, Peng X. Autophagy attenuates particulate matter 2.5-induced damage in $\mathrm{HaCaT}$ cells. Ann Transl Med 2021;9(12):978. doi: 10.21037/atm-21-2146
37. Hüls A, Sugiri D, Fuks K, et al. Lentigine Formation in Caucasian Women-Interaction between Particulate Matter and Solar UVR. J Invest Dermatol 2019;139:974-6.

38. Leonardi-Bee J, Ellison T, Bath-Hextall F. Smoking and the risk of nonmelanoma skin cancer: systematic review and meta-analysis. Arch Dermatol 2012;148:939-46.

39. Marrot L. Pollution and Sun Exposure: A Deleterious Synergy. Mechanisms and Opportunities for Skin Protection. Curr Med Chem 2018;25:5469-86.

40. Mujtaba SF, Dwivedi A, Yadav N, et al. Singlet oxygen mediated apoptosis by anthrone involving lysosomes and mitochondria at ambient UV exposure. J Hazard Mater 2013;252-253:258-71.

41. Piao MJ, Ahn MJ, Kang KA, et al. Particulate matter 2.5 damages skin cells by inducing oxidative stress, subcellular organelle dysfunction, and apoptosis. Arch Toxicol 2018;92:2077-91. 